\title{
Judging the location of features of naturalistic and geometric shapes
}

\author{
SHARON L. GREENE, EILEEN PLASTOW, and LILA G. BRAINE \\ Barnard College, New York, New York
}

\begin{abstract}
It is hypothesized that left-right orientation judgments always require judging the location of a feature relative to a framework, but that up-down judgments are often merely judgments of uprightness and nonuprightness (Braine, 1978). Three reaction time experiments examined the relative difficulties of up, down, left, and right judgments when they all explicitly required locating features relative to an external spatial framework. In the two experiments that utilized naturalistic figures, the locations of front features were identified when shapes were presented upright, upside down, or turned $90^{\circ}$. In the third experiment, the location of a dashed side of geometric shapes was identified. The results suggest that left-right feature-location judgments take slightly longer than up-down judgments when orientation cues are minimized by the use of symmetric geometric shapes. However, when naturalistic shapes are judged, these differences are either augmented or diminished, depending on the orientation of the stimuli.
\end{abstract}

The present work explores some factors affecting the relative difficulty of making left or right shape orientation judgments, as compared with making up or down judgments. Although traditional theories (e.g., Corballis \& Beale, 1976) have attributed this difference to problems in coding information along the horizontal, as opposed to vertical, axis, a number of recent studies do not support this interpretation (Braine \& Eder, 1983; Maki, Grandy, \& Hauge, 1979; Rock, Di Vita, \& Barbeito, 1981; Sholl \& Egeth, 1981; Vogel, 1984). An alternative view suggests that judgments of upright and upside down are easier because they can be made in cognitively more simple ways than judgments of left and right (Braine, 1978)

To judge a shape as turned $90^{\circ}$ to the left or right requires a description of the relation between a feature of the shape, for example, the top, and a side of the spatial framework (i.e., left or right). However, other orientation judgments can be made without locating a feature in relation to the spatial framework. A shape may be identified as looking upright or nonupright; this judgment of rightly turned or wrongly turned could be based on a match or mismatch between the input and information coded in memory. [This is assumed to be the basis for the normal-reversed judgment made in mental rotation

This study was supported by NICHD Grant HD-09513. We would like to thank Robert Proctor and Michael Corballis for helpful comments on an earlier version of the manuscript. The first author is currently at Bell Communications Research. The second author is at the University of Surrey, England. Reprints may be obtained from all three authors: Sharon L. Greene, Bell Communications Research, Morris Research \& Engineering Center, Rm. 2P-358, 435 South Street, Morristown, NJ 07960; Eileen Plastow, Department of Psychology, University of Surrey, Guilford, Surrey, England; or Lila G. Braine, Department of Psychology, Barnard College, 3009 Broadway, New York, NY 10027-6598. tasks using letters as stimuli (Cooper \& Shepard, 1973).] Nonupright shapes can be identified as upside down or sideways by noting whether the top-bottom axis of the figure is vertical or horizontal. Thus, when an object is wrongly turned and has a vertical top-bottom axis, a down judgment may be made without the need for relating features to a particular side of the spatial framework. These orientation judgments, although obviously applicable to letters and naturalistic shapes, also apply to nonrepresentational geometric shapes, since many have an apparent upright (e.g., Braine, 1978) and, as Rock (1973) claims, may be coded as having top, bottom, and side features.

A series of experiments with children and adults has shown that the upright-nonupright distinction is the easiest, the upside-down/sideways distinction is at an intermediate level of difficulty, and the left-right distinction is the most difficult (Braine, Greene, \& Plastow, 1984; Braine, Lerner, \& Relyea, 1980; Braine, Relyea, \& Davidman, 1981). If the main reason for the difference between leftright and up-down judgments is that the former are descriptions of locations whereas the latter are judgments of looking rightly turned or wrongly turned, then the question arises as to whether the two sets of judgments would be of equivalent difficulty if the task required that all judgments be location judgments.

The available literature on location judgments does not provide an answer to this question. Most studies have required judgment of the location of whole items (Farrell, 1979) or of the location of one item relative to another (Clark \& Chase, 1972; Just \& Carpenter, 1975). They have found that left-right judgments are usually, although not always (see Glushko \& Cooper, 1978, Maki et al., 1979, and Sholl \& Egeth, 1981), more difficult than topbottom judgments. The few studies that have purported to examine judgments of the location of a feature of an 
item, have used arrows in various orientations (Farrell, 1979; Maki et al., 1979; Sholl \& Egeth, 1981). Subjects judged that the arrow was pointing up, down, left, or right, or made discriminations between up-down- and between left-right-pointing arrows. However, while it has been assumed that these judgments were all made by describing the location of the head of the arrow, it is possible that the arrow pointing up may have been encoded as the upright orientation. The work mentioned above, indicating reliable judgments for the upright of geometric shapes (Braine, 1978), supports this possibility. This work, then, does not preclude the confounding of up, upside down judgments with rightly turned, wrongly turned cues.

To insure that some implicit coding of uprightness did not co-occur with the "up" location judgment, in the following experiments, using natural objects, subjects were required to locate an object's front features (of side views). So, for example, when front features were located in the "up" position, the object was actually turned sideways. By so doing, spatial judgments (up, down, left, and right) could be obtained without ever having the rightly turned cue co-occur with the "up" judgment, or the wrongly turned cue co-occur with the nonup judgments.

Figure 1 illustrates the stimuli. Judgments involving the location of front features will be referred to as location judgments, whereas those made with respect to the top-

\section{Up
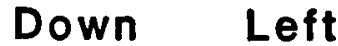 \\ Right}
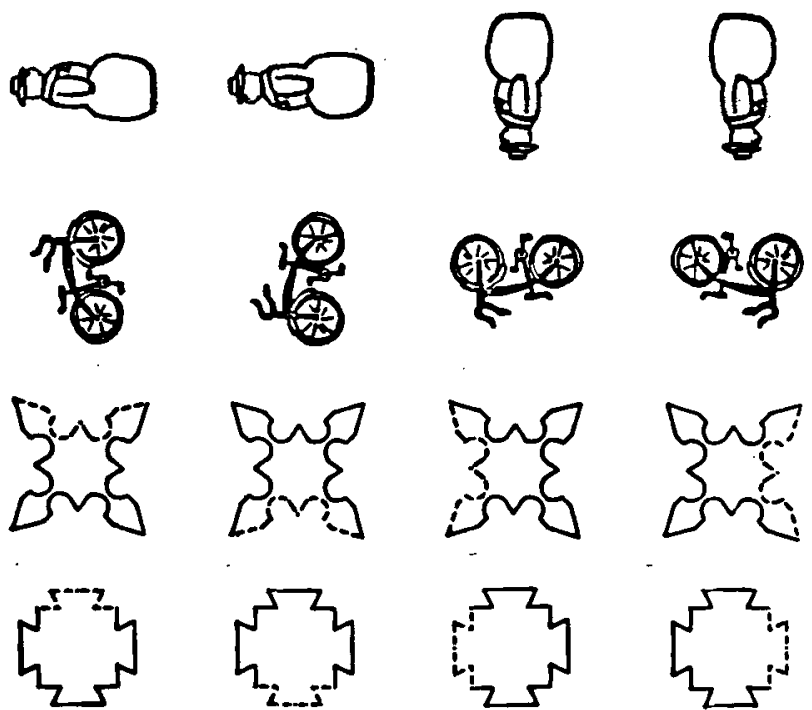

Figure 1. Samples of stimuli used in the three experiments. Experiments 1 and 2 utilized drawings of objects, such as the snowman and the bicycle, which were shown facing up, down, left, or right. In Experiment 1, the left- and right-facing stimuli were turned upside down, as shown above, whereas in Experiment 2 they were upright. Symmetric geometric shapes, with one side dashed, were used in Experiment 3. bottom axis of the figure will be referred to as orientation judgments (e.g., the figure is upside down, turned to the left, and so on). The feature-location response then always differs from a response based on orientation cues.

\section{EXPERIMENT 1}

This experiment examined RT patterns for judgments of the location of front features of natural objects. None of the objects were ever presented in the upright. When the fronts were facing up or down, the figures were rotated $90^{\circ}$ to the left from the upright. When the fronts were facing left or right, the figures were presented upside down (see Figure 1). A four-choice version of this task and a two-choice, two-condition version were run, in order to examine the effect of available response alternatives on judgment times.

\section{Method}

Subjects. Subjects in the four-choice task consisted of 13 female and 7 male right-handed college students between 17 and 30 years of age. They were drawn primarily from introductory psychology classes. Eight subjects were paid $\$ 5$ for their participation; the rest participated for extra credit. Data from a further 6 subjects were discarded, either because they persistently responded incorrectly on more than three items or because they had excessively long RTs, defined as a significant deviation from group performance. ${ }^{1}$ These criteria were used in all subsequent experiments.

Subjects in the two-choice task consisted of 10 female and 10 male right-handed summer-school students who were paid $\$ 4$ each to participate in the experiment. They ranged in age from 18 to 28 years. Data from six other subjects were discarded.

Apparatus and Stimuli. The stimuli were black line drawings of 20 familiar, mono-oriented shapes (see Figure 1). All the drawings were asymmetric side views. In the upright orientation, half faced left, half right. The drawings were of the following items: airplane, bicycle, bird, boot, car, chair, coat, doll, elephant, face, kangaroo, mouse, pig, rabbit, ship, shoe, snowman, teakettle, train, and truck. The long axes averaged $2.8 \mathrm{~cm}$, with a range from 2.3 to $3.5 \mathrm{~cm}$.

The stimuli were presented in a Gerbrands tachistoscope (Model T-3a). They appeared in the center of an $8 \times 8 \mathrm{~cm}$ square aperture, at a viewing distance of $81 \mathrm{~cm}$, for an exposure duration of 300 msec. A fixation point was shown during the interstimulus interval. The stimulus field was adjusted to an intensity of $.39 \mathrm{fL}$, and the fixation field, to .05 $\mathrm{fL}$ (as measured by a UDT Universal optometer). For the four-choice task, a Hunter Klockounter (Model 120c) timed the period from stimulus onset to the signal from a Lafayette voice relay (Model 1604A). This relay was activated by the subject's vocal response into a Sony microphone. For the two-choice task, and all subsequent experiments, the timing was done by an assembly language routine on a TRS-80 Model III computer. Calibration measurements showed that the computer and Hunter Klockounter agreed to within about $0.2 \%$.

Procedure. Subjects were initially shown a sheet displaying the full set of 20 drawings. In the four-choice task, each object was displayed with the four facing positions for that item in a row on the sheet. The four positions were: front facing up, front facing down, front facing left, and front facing right. Before the start of each condition in the two-choice, two-condition task, the subjects were shown all the objects in the two facing positions appropriate for the condition (up-down or left-right). Condition order was counterbalanced across subjects.

The subject's attention was drawn to the designated front of each object. The subject was then told that each of these pictures would 
be presented in the positions shown. In the four-choice task, they were told to respond as quickly and accurately as possible with the words "up," "down," "left," or "right," according to which way the front was facing. Forty items were given as practice, 10 in each facing position, followed by 160 experimental trials, each item being presented twice in each position. Stimuli to which errors were made were shown again after $40,80,120$, and 160 trials. There was a short rest after 80 trials.

In the two-choice, two-condition task, the subjects were to respond with "up" or "down" (up-down condition) and "left" or "right" (left-right condition). They were given 20 practice trials ( 10 in each of the two facing positions) followed by the 80 experimental trials for that condition-each item being presented twice in both facing directions. Any stimuli to which errors were made were shown again after 40 and after 80 trials. There was a short rest between conditions.

In all experiments, the stimuli were presented to each subject in a different random order with the following constraints: (1) The same facing position did not appear consecutively more than three times, (2) the four positions (for the four-choice task), or two positions (for the two-choice task) appeared with equal frequency in the first and second half of each set of 80 trials, and (3) the same item could not follow itself consecutively. Trials 80 to 160 were a repeat of the order for the first 80 trials in the four-choice task.

\section{Results and Discussion}

In an analysis of the four-choice task, a one-way ANOVA comparing the facing directions (up, down, left, and right) proved to be highly significant $[F(3,57)=$ 22.49 , $\mathrm{p}<.00001]$. The RTs to the left and right positions of the front were much longer than those to the up and down positions. The average values, in milliseconds, were as follows: up, 841; down, 861; left, 965; and right, 979 (see Figure 2). The differences between left and right and between up and down were nonsignificant (using Bonferroni $t$ tests), but all other such pairwise comparisons were significant $(p<.01,19 \mathrm{df})$. The average error rate was .01 (a total of 45 errors), and provided no evidence for a tradeoff between speed and accuracy.

The results from the two-choice, two-condition task were analyzed in a two-way ANOVA on condition order (up-down first vs. second), $\times$ condition (up-down vs. leftright). It yielded significance on the condition variable $[\mathrm{F}(1,18)=28.04, \mathrm{p}<.0001]$ and on the condition $\times$ order interaction $[F(1,18)=4.37, p<.05]$. As in the four-choice task, left and right judgments again took longer to make (averaging $660 \mathrm{msec}$ ) than up or down judgments (averaging $615 \mathrm{msec}$ ). The Bonferronit values were all significant, with the exception of the differences between up and down and between left and right $(p<.05,19 \mathrm{df})$. The error rate was low overall (.01). Errors were evenly distributed across the four responses.

When the up-down condition came first, up and down RTs were higher than when this condition came second. When the left-right condition came first, left and right RTs were higher than when this condition came second. This represents a normal practice effect, and accounts for the condition $x$ order interaction.

The difference between RTs to up- or down-facing stimuli and to left- or right-facing stimuli indicates that the left and right sides of space are harder to identify than the top and bottom sides. However, another interpreta- tion is possible if the orientation of a stimulus was an important determinant of RT. If the upside-down orientation of the stimuli that faced left or right was somehow more salient than the $90^{\circ}$ turn of the up- and down-facing stimuli, then the left and right judgments may have taken longer because of the effort to inhibit an upside-down response rather than because of any inherent difficulty in making left-right judgments.

The error data in the four-choice task support the possibility that orientation was processed, even though orientation information could only interfere with the appropriate response. With the exception of the up errors (four of the five were to down), the other errors tended to be to the orientations of the figures (as opposed to the way they faced). Thus, when errors were made to the upside-down figures with the front facing left or right, the incorrect judgment most often made was down. Errors to the left-turned figures with fronts facing down were most often incorrectly identified as left. This indicates a conflict between making the judgment on the basis of front feature location and making it on the basis of the object's orientation.

Competition from a response to orientation cues should have been reduced in the two-choice, two-condition task, relative to the four-choice task, since in the former the conflicting orientation response was not an acceptable response alternative. When a stimulus faced left or right in the left-right condition, "down" (i.e., the stimulus orientation) was not a response alternative. Similarly, in the up-down-facing condition, "left" (the stimulus orientation) was not a response alternative. This may account in part for the reduction in RTs from the four-choice task to the two-choice task. ${ }^{2}$ However, stimulus orientation may have been processed even though the orientation response was not an alternative within a condition. If down were more salient than left, such processing still could be a factor in the lengthening of the left-right RTs relative to the up-down RTs.

\section{EXPERIMENT 2}

Response competition from orientation cues may have been a primary cause for the differences between left and right RTs and up and down RTs in Experiment 1. If so, left-right-facing judgments might be affected by a change in the orientation of the stimuli. On the assumption that the upright is the most natural orientation, it was hypothesized that orientation processing (and hence response competition) would be reduced by the presentation of left- and right-facing stimuli in the upright. Since, as before, front features of side views were to be located, all judgments still differed from judgments based on upright-nonupright cues. As in Experiment 1, a fourchoice version and a two-choice, two-condition version of this task were run.

\section{Method}

Subjects. Seventeen female and three male right-handed students between 17 and 21 years of age participated in the four-choice ver- 
sion of the experiment. Data from one further subject were discarded due to very long RTs. In the two-choice, two-condition version, the 16 female and 4 male (all right-handed) subjects ranged in age from 19 to 21 years. Data from an additional subject were discarded due to excessively long RTs. All subjects were recruited from introductory psychology courses and participated for extra credit.

Apparatus and Stimuli. The apparatus and stimulus drawings were identical to those used in the previous experiment.

Procedure. The general procedures for both the four-choice and two-choice tasks were the same as those used in Experiment 1 for the comparable tasks. While the stimulus drawings were the same as those used before, here the left- and right-facing stimuli were shown oriented in the upright, as opposed to the upside-down orientation (see Figure 1). In the two-choice task, half of both the upand down-facing figures were turned $90^{\circ}$ to the left from the upright; the other half were turned right. As before, the subject's task was to say which way the front was facing. The number of trials and stimulus ordering constraints were the same as in the prior experiment.

\section{Results and Discussion}

The one-way ANOVA on facing directions in the fourchoice task yielded a nonsignificant value of $F(3,57)=$ $1.34, \mathrm{p}>.20$. The average RT values, in milliseconds for the four judgments were as follows: up, 683; down, 675; left, 694; and right, 678 (see Figure 2). In contrast to the findings from Experiment 1, none of the differences between pairs of orientations was significant. The overall error rate was .01 , and although more errors were made to up-down- than left-right-facing stimuli, the difference was predominantly due to one item (as discussed below), indicating no general speed-accuracy tradeoff.

The advantage of up-down judgments over left-right judgments observed in Experiment 1 was not apparent in this four-choice task. Several factors are likely to have facilitated the left-right judgments relative to up-down judgments. First, the left- and right-facing judgments were naturally occurring ones. Side views of the objects used are common, and are likely to face left or right more often than up or down. Second, a necessary prerequisite for making the location judgment is identification of the front features. If front-feature identificaion is facilitated by the upright orientation, this prerequisite step would be faster for the left-right-facing stimuli than for the up-downfacing stimuli. This is supported by the pattern of errors observed. More errors were made to stimuli facing up or down (32 errors) than to those facing left or right (15 errors) $[t(19)=3.10, p<.05]$. The bicycle was responsible for most of these up and down errors. Sixteen of the 32 up-down errors were made on the bicycle, whereas only 3 of the 15 left-right errors were made on this item. The bicycle had a clear front-back axis but had front features that were difficult to distinguish, especially in nonupright orientations (see Figure 1). The fact that the accuracy of judging the bicycle in the up- and down-facing positions was much lower than in the left or right, supports the interpretation that the upright orientation aids identification of the front features.

Although Experiment 2 was designed to reduce incidental processing of orientation (by eliminating the possibly salient "down", orientation of left-right-facing stimuli), it did not succeed in this goal. The error data of the present four-choice task indicate that the orientations of the items were still processed. Although 14 of the 22 down errors were to up, 13 of these were due to the bicycle. Axis errors could be expected on this item, as discussed above (also see Figure 1). Excluding the bicycle, 7 of the 9 remaining errors on down stimuli were to "left," the way these stimuli were turned. The left- and right-facing stimuli were in the up orientation, and two-thirds of the errors to those stimuli were to up (independent of whether or not errors due to the bicycle are included). The exception to this pattern were the errors to up-facing stimuli, which were predominantly to down ( 9 out of 10 if one includes bicycle errors, and 6 out of 7 otherwise). This error pattern was precisely the same as that observed in Experiment 1, in which up errors were axis errors, but the errors to the non-up-facing stimuli were to the orientation to which the items were turned.

While processing of orientation in Experiment 1 could only interfere with correct responding, in the present fourchoice task an upright-nonupright distinction could have aided in the determination of feature location. Since an upright-nonupright distinction is easily made (Braine et al., 1980; Braine et al., 1981), it could have served to reduce the number of sides the subject had to attend to from four to two. For stimuli perceived as upright, a subject had to attend only to the left and right sides to determine where the front was located. For nonuprights, only the top and bottom sides required attention. If this four-alternative task had been transformed, cognitively, into essentially two two-choice tasks, one would expect lower RTs in this experiment than in the four-choice task of Experiment 1, since two-choice tasks are typically easier than four-choice tasks (e.g., Braine et al., 1984). The explicit two-choice, two-condition version of Experiment 2 did further reduce the overall RTs from the level of the four-choice task, but the reduction was not as great as the comparable reduction in Experiment 1 (see Figure 2).

Like the four-choice task, the two-choice task yielded no indication that left- and right-facing judgments were more difficult than up and down judgments. In fact, the RTs in the left-right-facing condition (averaging $575 \mathrm{msec}$ ) were lower than in the up-down-facing condition (averaging $614 \mathrm{msec}$ ). A two-way ANOVA on condition order (up-down first vs. second) $\times$ condition (updown vs. left-right), yielded a significant $F(1,18)=$ $10.54, \mathrm{p}<.005$, for the condition variable. Within a condition, left RTs did not differ from right RTs and up RTs did not differ from down RTs. The error rate was low (less than .01 over all trials). There was no evidence for a speed-accuracy tradeoff. The number of errors made in the up-down condition ( 11 errors) was not significantly different from the number of errors in the left-right condition ( 3 errors). However, as in the four-choice task, the bicycle was responsible for 5 of the 11 up or down er- 
rors, but only 1 of the 3 left or right errors. This supports the interpretation that front features are more easily discriminated when an object is upright than when it is nonupright.

While these data certainly argue against the left-right judgment being harder than the up-down judgment, this significant difference in RTs across conditions was really due to an unusual order effect. Examining the RT values for up-down versus left-right, when each of those conditions was the first condition experienced (a betweensubjects comparison), the RT differences were nonsignificant: $594 \mathrm{msec}$ for up-down and $585 \mathrm{msec}$ for left-right. However, when both were the second conditions experienced, the difference was significant. The average RT for the up-down condition was $633 \mathrm{msec}$, and for the leftright condition, $564 \mathrm{msec}[\mathrm{t}(18)=2.68, \mathrm{p}<.02]$. This means that RTs to the left- and right-facing stimuli were faster after experience on the up-down condition, as to be expected from practice on the task. Yet RTs to the upand down-facing stimuli were elevated as a result of practice on the left-right condition, relative to the values obtained when the up-down condition came first. There is no obvious explanation for this negative transfer.

Since the results from both four-choice tasks in Experiments 1 and 2 suggest that orientation is being processed even when not called for, it is useful to examine the effect of a figure being turned upside down, instead of upright, when a left- or right-facing judgment is required. This may be done by comparing RTs from the left-right condition in the two-choice task of Experiment 1 with those in Experiment 2. In both cases, to avoid contamination by differences in practice effects, only the data from subjects having this condition first are examined. It is clear that the left- and right-facing judgments were impeded by having the stimuli presented turned upside down. On the average, these judgments were $81 \mathrm{msec}$ slower when the stimuli were upside down than when they were upright $[\mathrm{t}(18)=2.59, \mathrm{p}<.02]$. A comparable test for differences in RTs in the up-down judgments between the two experiments was nonsignificant.

\section{EXPERIMENT 3}

The data from Experiment 1 indicated that left- and right-facing judgments were slower than up and down judgments when no stimuli were in the upright. This difference was maintained, although substantially reduced, even when up and down judgments were separated in time from left and right judgments. This difference did not appear when the left- and right-facing stimuli were in the upright in Experiment 2. Both previous experiments indicate that orientation is processed even though such processing may retard the required judgment. The present experiment assesses the difficulty of feature location judgments of geometric shapes constructed to eliminate any possible cues related to orientation (such as differences in lengths of the main axes).

\section{Method}

Subjects. Twelve female and eight male subjects participated in the experiment. All subjects were right-handed. They ranged in age from 18 to 26 years. They were summer-school students and were each paid $\$ 4$ for participating.

Apparatus and Stimuli. The same apparatus was used as in the prior experiments. The stimuli consisted of 20 computer-generated line-drawings (see Figure 1). Each stimulus was a different geometric shape constructed to be symmetrical around the vertical, horizontal, and diagonal axes. The location judgment was to be made based on which of the four sides, up, down, left, or right, was dashed instead of solid. The shapes varied in complexity, from very simple (e.g., a square) to more complex, with several turns on each side. They ranged in size from 2.5 to $5.1 \mathrm{~cm}$ along any side. This approximated the size range of the stimuli from the prior experiments. Half of the shapes were made of straight lines only, and half were made of at least some curved lines.

Procedure. The subjects were to identify the location of the dashed side by saying "up," "down," "left," or "right." All other aspects of the procedure were identical to those from the prior four-choice tasks.

\section{Results and Discussion}

The RTs indicate that left and right judgments (averaging $643 \mathrm{msec}$ ) again were longer than up or down judgments (averaging $605 \mathrm{msec}$ ). A two-way ANOVA on location (up, down, left, or right) and shape (straight lined or curved) yielded a significant $F(3,19)=16.56$, $p<.0001$, for location only. The Bonferroni t tests between pairs of location judgments indicate that left was not significantly different from right, nor was up from down. All other remaining comparisons but that between down and left reached statistical significance $(p<.05$, $19 \mathrm{df}$ ). The longer RTs for left and right than for up and down (see Figure 2) is support for the notion that the left and right sides of the spatial framework are harder to discriminate than the top and bottom sides.

The RTs obtained in all three experiments are summarized in Figure 2. They show the effects of two main factors, orientation and number of response alternatives, on the time to judge the location of a feature. Orientation of the stimuli played a role in several ways. Judgments of left-right location were faster when the shapes were upright rather than upside down. This effect is evident in the comparison of the two-choice tasks of Experiments 1 and 2 (when the subject really needed to attend only to the ends of the horizontal axis regardless of the orientation of the stimulus). It also appears in the more complex four-choice tasks of Experiments 1 and 2. In addition, uprightness (of the stimuli with fronts at the left and right) had a possible further effect on location judgments within the four-choice version of Experiment 2 by providing a basis for simplifying the task from four choices to two.

The second main factor affecting RT was the number of choices available, as can be seen in the comparison of the four-choice and two-choice, two-condition tasks in Experiments 1 and 2 . The effects of orientation and number of choices combined, in the four-choice task of Experiment 1 , to produce the highest RTs. 


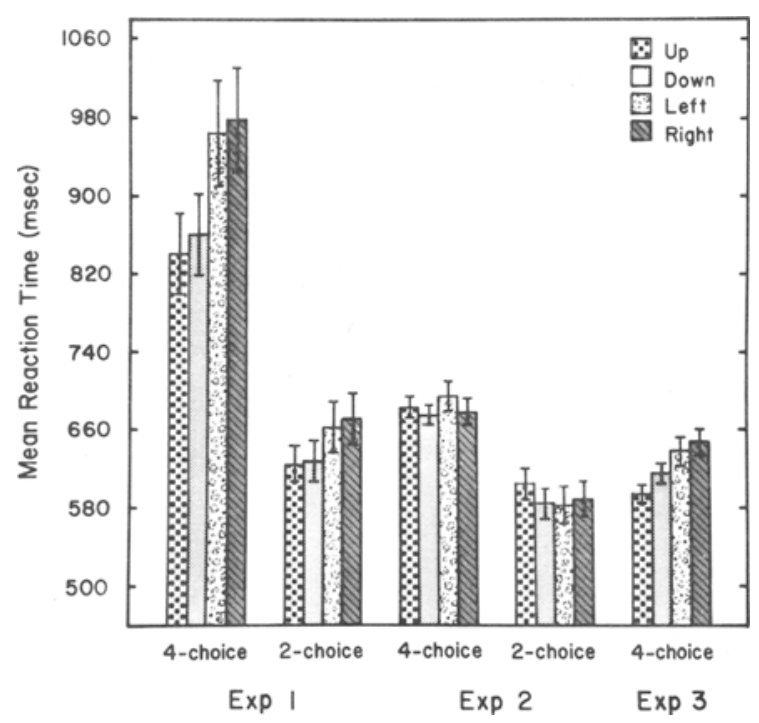

Figure 2. Means and their standard errors (in milliseconds) for each of the location judgments in Experiments 1-3. The two-choice, two-condition versions of Experiments 1 and 2 show data from first conditions only; up and down means are from the 10 subjects who had the up-down condition first, and left and right means are from the other 10 who had the left-right condition first. All judgments in the four-choice tasks of Experiments 1-3 were made in one condition; the means are based on all 20 subjects. In Experiment 1, leftand right-facing stimuli were turned upside down. They were upright in Experiment 2. Geometric shapes were used in Experiment 3.

\section{GENERAL DISCUSSION}

Earlier work (Braine, 1978; Braine et al., 1984) had indicated that left-right orientation judgments were based on locating features of an object in an external spatial framework, whereas up-down judgments could be based on cognitively simpler processes. Forcing subjects to make up-down judgments based on a feature-location strategy would lead to judgment times equal to those for left-right judgments provided the left and right sides of the spatial framework were no more difficult to identify than the top and bottom sides. However, the results of Experiment 3 indicate that it is slightly harder to judge the location of features with respect to the left or right sides of the spatial framework than it is to judge their location with respect to the top or bottom sides. This effect is small, but reliable, and is consistent with a wide range of other studies of location using geometric stimuli and spatial descriptions (e.g., Farrell, 1979; Maki et al., 1979).

Perhaps more important, though, are the findings that this slight left-right feature-location difficulty can be overshadowed by other factors when more natural stimuli are being judged. The first two experiments indicate that when these stimuli are used, the difficulty of a location judgment cannot be easily disentangled from factors related to stimulus orientation. Error data and RT differences between experiments emphasize how RT patterns for a location task can be altered simply by changing the orientation of the stimuli judged, keeping all else constant.
Automatic processing of orientation, in and of itself, does not explain why the left-right feature-location judgments were slower when stimuli were upside down than when they were upright. An interaction between feature location and stimulus orientation must have occurred. A conceptual breakdown of the feature-location task into two steps suggests a vehicle for this interaction. The first step requires identification of the specific features that constitute the shape's front. The second step requires a spatial judgment; describing the relation between these features and a part of the spatial framework.

On the geometric stimuli, the feature relevant for the location judgment (dashed line) was constant across shapes. A subject first had to locate the dashed side on the shape, but this initial step should not have differed for the different locations unless some selective scanning or attentional strategies had been used. Barring such strategies, it is assumed that the differences in RTs observed resulted from the spatial location step of the judgment.

For the naturalistic stimuli, a different front feature had to be found for each item before the location judgment could be made. It is hypothesized that the processing necessary to locate the front features on the shape itself varies with a figure's orientation, being easiest when the object is upright. The lower RTs in left-right featurelocation judgments of upright stimuli, relative to those of upside-down stimuli, would then be a function of processes in the front feature-identification step, not the spatial location step of the judgment.

It is possible that front feature identification is linked to object identification. The literature is mixed regarding the relationship between object identification and orientation. Some studies using simple visual stimuli (usually alphanumeric characters) have not shown object identification to vary as a function of orientation (Corballis, Zbrodoff, Shetzer, \& Butler, 1978, Experiment 2; Eley, 1982; White, 1980). However, even if object identification does not always vary systematically across nonupright orientations, the upright usually maintains a privileged position (Corballis et al., 1978, Experiments 1 and 3; Eley, 1982, Experiment 1; Simion, Bagnaro, Roncato, \& Umilta, 1982, Experiment 3). Clear evidence for the advantage of the upright, as well as systematic variation in object identification as a function of object orientation, were demonstrated by Jolicoeur and Landau (1984). Similarly, when naturalistic objects are used, the upright advantage is found consistently (Braine, 1965; Gibson \& Robinson, 1935; Rock, 1973; Shwartz, 1981).

Further research is needed to examine the relationship between orientation, front feature identification, and object identification. However, if the identification of front features is easier in the upright or is tied to object identification, and the latter is fastest for uprights, then the equality of RTs across the four judgments in Experiment 2 may be understood. The identification of front features of the left- and right-facing stimuli would be facilitated, relative to the up- and down-facing stimuli, since the former were upright. This left-right advantage in fea- 
ture identification would cancel any slight difficulty inherent in the left-right spatial location judgment. Similarly, any difficulty in front-feature identification that occurred when the left- and right-facing stimuli were upside down (Experiment 1 ) would add to any already existing difficulty in identifying the location of features in the left or right sides of the framework.

The present findings, then, have highlighted the dependence of judgments of feature location on the processing of shape and orientation. The use of naturalistic figures has revealed the richness of processing underlying such judgments. This is no reason, however, to assume that this processing is limited to naturalistic figures. The geometric shapes used in the present experiment were symmetric around both major axes and around the diagonals; they were constructed explicitly to prevent the perception of uprightness and to avoid asymmetries that might influence the difficulty of finding the relevant features. The so-called "pure" judgments of feature location observed in Experiment 3 may occur for only this relatively narrow range of shapes. By systematically constructing geometric shapes to display apparent uprightness and other attributes of naturalistic shapes (Braine, 1978; Howard, 1982; Schaller \& Harris, 1975), the interactions between orientation cues and location judgments may be further elucidated.

\section{REFERENCES}

BraINe, L. G. (1965). Disorientation of forms: An examination of Rock's theory. Psychonomic Science, 3, 541-542.

BraINE, L. G. (1978). A new slant on orientation perception. American Psychologist, 33, 10-20.

Braine, L. G., \& EdER, R. A. (1983). Left-right memory in 2-yearold children: A new look at search tasks. Developmental Psychology, 19, 45-55.

Braine, L. G., Greene, S. L., \& Plastow, E. (1984, April). Orientation perception: A matter of contrasts. Paper presented at the meeting of the Eastern Psychological Association, Baltimore.

Braine, L. G., LeRner, C., \& Relyea, L. (1980). Levels in the identifying of orientation by preschool children. Journal of Experimental Child Psychology, 30, 171-185.

Braine, L. G., Relyea, L., Davidman, L. (1981). On how adults identify the orientation of a shape. Perception \& Psychophysics, 29, 138-144.

Clark, H. H., \& Chase, W. G. (1972). On the process of comparing sentences against pictures. Cognitive Psychology, 3, 472-517.

CoOper, L. A., Shepard, R. N. (1973). Chronometric studies of the rotation of mental images. In W. G. Chase (Ed.), Visual information processing. New York: Academic Press.

Corballis, M. C., \& BEALE, I. L. (1976). The psychology of left and right. Hillsdale, NJ: Erlbaum.

Corballis, M. C., Zbrodoff, N. J., Shetzer, L. I., \& Butler, P.
B. (1978). Decisions about identity and orientation of rotated letters and digits. Memory \& Cognition, 6, 98-107.

ELEY, M. G. (1982). Identifying rotated letter-like symbols. Memory \& Cognition, 10, 25-32.

FARRELL, W. S., JR. (1979). Coding left and right. Journal of Experimental Psychology: Human Perception and Performance, 5, 42-51.

Gibson, J. J., \& Robinson, D. (1935). Orientation in visual perception: Recognition of familiar plane forms in different orientations. Psychological Monographs, 46(Whole No. 210), 39-47.

Glushko, R. J., \& CoOPER, L. A. (1978). Spatial comprehension and comparison processes in verification tasks. Cognitive Psychology, 10, $391-421$.

HowARD, I. P. (1982). Human visual orientation (pp. 523-525). New York: Wiley.

JolicoeUR, P., \& LANDAU, M. J. (1984). Effects of orientation on the identification of simple visual patterns. Canadian Journal of Psychology, 38, 80-93.

Just, M. A., \& CARPenter, P. A. (1975). The semantics of locative information in pictures and mental images. British Journal of Psychology, 66, 427-441.

MakI, R. H., Grandy, C. A., \& Hauge, G. (1979). Why is telling right from left more difficult than telling above from below? Journal of Experimental Psychology: Human Perception and Performance, $5,52-67$.

Rock, I. (1973). Orientation and form. New York: Academic Press.

Rock, I., Di Vita, J., \& BARbeito, R. (1981). The effect on form perception of change of orientation in the third dimension. Journal of Experimental Psychology: Human Perception and Performance, 7, 719-732.

SCHAller, M. J., \& Harris, L. J. (1975). "Upright" orientations of forms change with subject age and with features of form. Perception \& Psychophysics, 17, 179-188.

SHOLL, M. J., \& EGETH, H. E. (1981). Right-left confusion in the adult: A verbal labeling effect. Memory \& Cognition, 9, 339-350.

SHWARTZ, S. P. (1981). The perception of disoriented complex objects. Unpublished manuscript.

Simion, F., Bagnara, S., Roncato, S., \& Umitta, C. (1982). Transformation processes upon the visual code. Perception \& Psychophysics, 31, 13-25.

VOGEL, J. M. (1984). Developmental changes in speed of encoding leftright orientation. Manuscript submitted for publication.

WHITE, M. J. (1980). Naming and categorization of tilted alphanumeric characters do not require mental rotation. Bulletin of the Psychonomic Society, 15, 153-156.

\section{NOTES}

1. The RT criterion used was based on the number of RTs greater than 1,000 msec. It was such that the numbers of these RTs exhibited by excluded subjects was more than 2.5 standard deviations above the mean number of such RTs produced by included subjects.

2. Even if the RTs of the six subjects excluded from analyses due to excessively long RTs were included, the resulting mean of the RTs in the two-choice task would still be $245 \mathrm{msec}$ lower than the mean of the four-choice task.

(Manuscript received July 16, 1984; revision accepted for publication January $29,1985$. 